(C) 1977 IEEE. Personal use of this material is permitted. However, permission to reprint/republish this material for advertising or promotional purposes or for creating new collective works for resale or redistribution to servers

or lists, or to reuse any copyrighted component of this work in other works must be obtained from the IEEE.

IEEE Transactions on Nuclear Science, Vol.MS-24, No.3, June 1977

\title{
SYNCHRONIZATION OF THE ZERO GRADIENT \\ SYNCHROTRON (ZGS) AND THE FAST-CYCLING BOOSTER II INJECTOR *
}

\author{
A. V. Rauchas \\ Argonne National Laboratory \\ Argonne, Illinois 60439
}

\section{Summary}

The planned method of booster injection into the ZGS requires the stacking of two booster beam bunches into the radial betatron phase space of the ZGS and necessitates precise synchronization of the two accelerators during the injection period. The requirement of maintaining independent radio-frequency (RF) steering control does not allow the frequencies of both accelerators to be locked. A synchronizer has been developed which detects a coincidence zero crossing of the two RFs, anticipates the next coincidence, and at that time, generates trigger pulses for the booster extraction and ZGS injection systems. It also maintains control of the sequence in which the total phase space area of the ZGS is loaded. Synchronizer accuracy is obtained by using high speed digital logic circuits. Design considerations and system operation are discussed.

\section{Introduction}

As part of the ZGS intensity improvement program, the construction of a $500 \mathrm{MeV}$, fast-cycling, booster injector synchrotron is nearing completion. The booster is designed to accelerate $>3 \times 10^{12}$ protons per pulse at a repetition rate of $30 \mathrm{~Hz}$. The ZGS RF accelerating system will be operated on the fourth harmonic and each RF bucket will be loaded with two booster bunches.

The ZGS magnetic field will begin rising from its nominal rest field value $\approx 0.27 \mathrm{kG}$. When the field reaches $1.69 \mathrm{kG}$, it will be held fixed at that value for $300-400 \mathrm{~ms}$. During this time, injection will occur. Each booster bunch will be extracted by a fast kicker magnet and transported to the ZGS. The bunch will be injected into the first ZGS long straight section ( $L 1$ ) and will coast three-eighths of a revolution to the second short straight section (S2). The bunch will be injected at a slight positive angle and, since the radial betatron tune during the whole injection period will be held constant at 0.833 , it will arrive in $\mathrm{S} 2$ at $90^{\circ}$ of betatron phase. There, with its size at a minimum and divergence at a maximum, the bunch will be kicked by the injection kicker magnet ${ }^{2}$ to make the divergence zero. This bunch will then coast on the equilibrium orbit with no betatron oscillations--missing the inflector--until three machine revolutions before the next booster bunch is injected to $\mathrm{S} 2$. At that time, the resident bunch will be bumped by the injection kicker to produce a small betatron oscillation. When the new booster bunch is injected, both bunches will be kicked so that the betatron oscillations of each bunch are equal and the net

WWork supported by the U. S. Energy Research and Development Administration. amplitude is zero. This process will be repeated for the other three ZGS buckets. Fig. 1 shows a phase space diagram illustrating the injection process.

\section{Synchronizer Design Considerations}

During injection, the ZGS RF system will operate at $5.30 \mathrm{MHz}$. The booster, with a radius one-fourth that of the ZGS and operating on the first harmonic, will have an extraction frequency of $5.29 \mathrm{MHz}$. Because of the large radial aperture of the $Z G S$, the equilibrium orbit could be shifted to the outside, so the two RF systems could be phase locked and synchronization achieved rather easily. This method was not used because of the requirement of maintaining independent $\mathrm{RF}$ steering control. The synchronization method selected was to detect coincidence zero crossings of the two RFs when enabled by certain machine conditions. The time of the next coincidence with the same conditions can be anticipated and the booster extraction and ZGS injection systems triggered at the proper time.

Since the booster bunch length is the same as the length of the ZGS phase space, accuracy of the coincidence detector and timing circuits is of utmost importance for efficient injection. A coincidence window of $3 \mathrm{~ns}$ was selected as a maximum allowable phase difference of the two RF zero crossings. Under these conditions, coincidences would occur for eight ZGS RF cycles after which the phases would exceed the coincidence window for $98 \mu \mathrm{s}$.

If an acceptable coincidence occurred during the first set, injection would occur during the second set of coincidences. Since the booster beam will match the acceptance of the ZGS for only $200 \mu \mathrm{s}$, injection will have to occur within that time. If the proper conditions are not met, that booster pulse will be aborted and an attempt will be made during the next pulse.

The ZGS injection kicker magnet is made up of three sections, each with its own strip line and firing circuit. The first fill of a ZGS bucket will require the firing of all three sections. For the second fill, a single section will be fired, followed in approximately $3 \mu \mathrm{s}$ by firing of the other two. The synchronizer will have to keep track of the filling sequence so that the proper strip lines are charged and fired.

\section{Synchronizer Operation}

During each booster cycle, two pulses will be sent to the synchronizer. The first, "Booster Ready," will occur when the booster has accelerated enough beam above a preset threshold to extraction energy. The other pulse, "Booster Sync," will occur $1 \mathrm{~ms}$ after the booster peak field is attained. 
The synchronizer will be enabled by the 7GS programmer at the beginning of the $1.65 \mathrm{kG}$ "front porch." The next "Booster Sync" pulse will trigger the injection kicker charging circuits to charge for a first fill. Feedback from the charging circuits will notify the synchronizer when charging is complete and arm the coincidence detector circuitry. The following "Booster Ready" pulse will enable the coincidence detector. An ultrahigh speed analog comparator will be used as a zero-crossing detector for each of the two RF signals. The se signals will originate from RF pickups at the respective RF cavities and will be buffered and delayed to compensate for cable lengths and the flight time of the protons from the booster to the $Z G S$. The coincidence detector circuitry will produce a pulse each time the two RF zero-crossings are within $3 \mathrm{~ns}$ of each other.

Two shift registers will be used to track the filled ZGS buckets. Although they both are 8-bit shift registers, each is wired in a recirculating 4-bit mode. The other bits are not used, but are provided for any future change in the $Z$ GS RF harmonic. Both registers will be clocked at the ZGS accelerating voltage frequency, so each RF bucket will have a corresponding bit in each shift register. One register will store first fill information, the other, second fill information. As each booster bunch is injected into the ZGS, a logical "one" will be loaded into the appropriate bit. The status of these shift registers will determine if a coincidence is acceptable. For a first fill, both corresponding bits will have to be at a logical "zero." A second fill will require the first fill bit to be at a logical "one" with the other register's bit at a logical "zero." Since the coincidences will occur in a burst of eight at the ZGS RF rate, and this corresponds to two revolutions around the machine, an acceptable coincidence will occur during the burst. That particular coincidence will then trigger the timing circuitry of the synchronizer.

The timer will be a $100 \mathrm{MHz}$ decade counter, capable of counting to $99.9 \mu \mathrm{s}$. All output trigger pulses will be generated by high speed digital comparators, preset by front panel digital switches. The comparators will provide a resolution of $100 \mathrm{~ns}$. Additional resolution will be provided by delaying the comparator output by two sets of programmable delay lines; one set to provide 10 ns delay steps, the other, I ns delay steps.
Five trigger pulses will be generated:

$\begin{array}{ll}\text { First Fill } & \text { 1. Booster Kicker Fire } \\ & \text { 2. ZGS Kicker Fire } \\ \text { Second Fill } & \text { 3. ZGS Kicker Bump Fire } \\ & \text { 4. Booster Kicker Fire } \\ & \text { 5. ZGS Kicker Fire }\end{array}$

Provisions for additional triggers will be provided for any future equipment requiring precise triggering during the injection period.

After a booster bunch is loaded into the $\mathrm{ZGS}$ and injection has not been terminated, the next "Booster Sync" pulse will trigger the proper injection kicker charging circuits for the next fill. Injection will be terminated when all ZGS buckets are full or the end of the front porch is reached. With the booster operating at $30 \mathrm{~Hz}$, the ZGS will normally be filled in $267 \mu \mathrm{s}$. Having a longer front porch will allow additional attempts at filling the ZGSbuckets if any failure in the booster extraction or ZGS injection systems occurs.

Internal test circuitry will be included to help in diagnosing any synchronizer logic failures. In this mode, the synchronizer will isolate itself from all inputs and outputs and slowly step through a hypothetical injection sequence. All steps will be displayed by lights, allowing an operator to quickly diagnose any problem. The synchronizer control panel is shown in Fig. 2 and an operational flow chart in Fig. 3.

\section{Acknowledgements}

The author wishes to thank D. Suddeth and J. Bogaty for their suggestions during the initial design of the synchronizer. Also, W. Chyna and W. Czyz for their work in the construction.

\section{References}

1. Booster II, A $500 \mathrm{MeV}$ Booster Injector For The Zero Gradient Synchrotron - A Design Report, internal publication.

2. D. E. Suddeth, R. L. Kustom and D. R. Schmitt, "Zero Gradient Synchrotron Booster Injection," IEEE Transactions on Nuclear Science (June, 1975), Vol. NS-22, No. 3, p. 1488. 


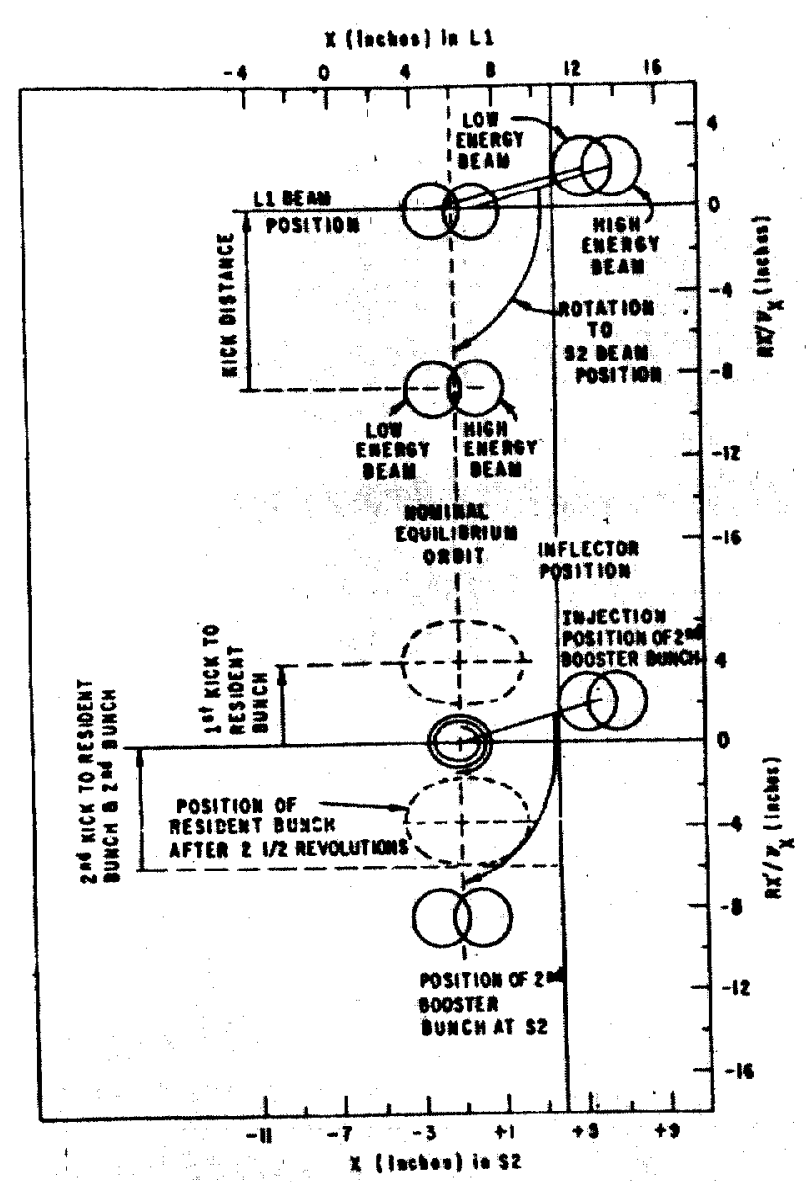

Fig. 1.

Phase space diagram of injection process.

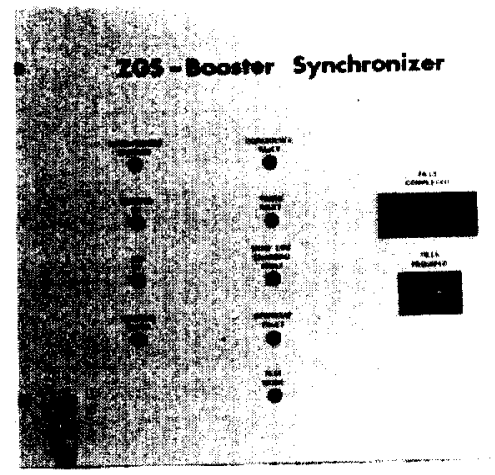

Fig. 2 .

Synchronizer control panel.

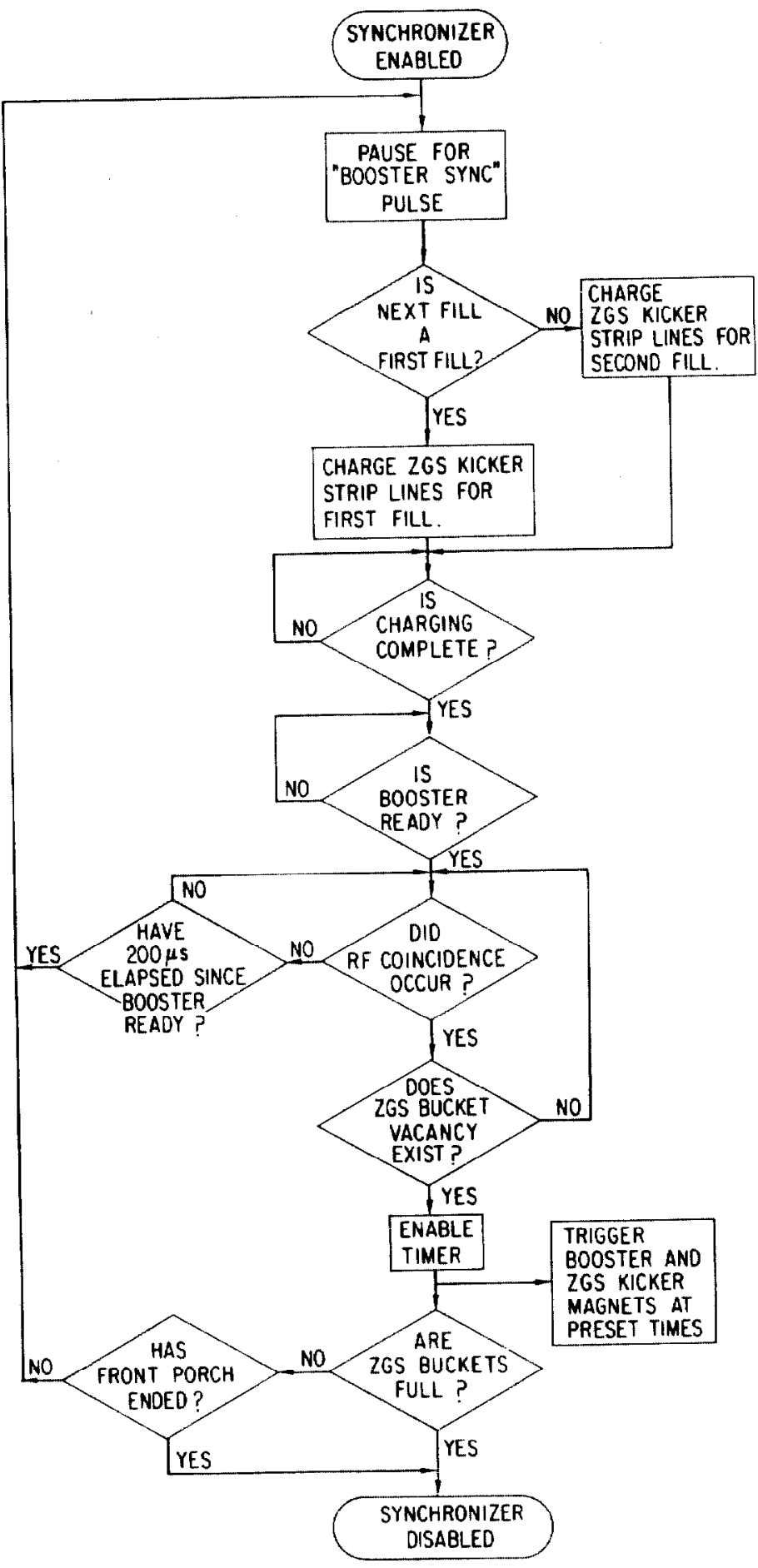

Fig. 3.

Synchronizer flow chart. 